\title{
Dust crystallinity in protoplanetary disks: the effect of diffusion/viscosity ratio
}

\author{
Ya. Pavlyuchenkov and C. P. Dullemond
}

\author{
Max Planck Institut für Astronomie, Königstuhl 17, 69117 Heidelberg, Germany \\ e-mail: pavyar@mpia.de
}

Received 16 February 2007 / Accepted 27 May 2007

\begin{abstract}
The process of turbulent radial mixing in protoplanetary disks has strong relevance to the analysis of the spatial distribution of crystalline dust species in disks around young stars and to studies of the composition of meteorites and comets in our own solar system. A debate has gone on in the recent literature on the ratio of the effective viscosity coefficient $v$ (responsible for accretion) to the turbulent diffusion coefficient $D$ (responsible for mixing). Numerical magneto-hydrodynamic simulations have yielded values between $v / D \simeq 10$ (Carballido et al. 2005, MNRAS, 358, 1055) and $v / D \simeq 0.85$ (Johansen \& Klahr 2005, ApJ, 634, 1353). Here we present two analytic arguments for the ratio $v / D=1 / 3$ which are based on elegant, though strongly simplified assumptions. We argue that whichever of these numbers comes closest to reality may be determined observationally by using spatially resolved mid-infrared measurements of protoplanetary disks around Herbig stars. If meridional flows are present in the disk, then we expect less abundance of crystalline dust in the surface layers, a prediction which can likewise be observationally tested with mid-infrared interferometers.
\end{abstract}

Key words. accretion, accretion disks - radiative transfer - diffusion

\section{Introduction}

Turbulent mixing plays an important role in the physics of protoplanetary accretion disks. The same turbulence that is responsible for the anomalous viscosity of the disk (and thus for the accretion process) is also responsible for the radial and vertical mixing of material in the disk. This mixing is likely to have strong influence on thermochemical processes and grain growth in disks. For instance, models of the gas-phase chemistry in disks turn out to be strongly dependent on the level of turbulent mixing (Gammie 1996; Semenov et al. 2004; Ilgner 2006). This is because some chemical reactions may be very slow in some regions but fast in another. Thus mixing can transport processed gas from the latter region to the former region, affecting the molecular abundances there.

For dust solid-state chemistry mixing plays presumably an even bigger role. Some evidence from meteoritics points to weak mixing, such as the recently discovered chemical complementarity of chondrules and matrix in some chondrites (Klerner \& Palme 2000). On the other hand, the existence of CalciumAluminium-rich Inclusions (CAIs) in many chondritic meteorites points toward some level of mixing in the early solar system, since CAIs are thought to form closer to the sun. Samples returned from comet Wild 2 by the STARDUST mission revealed that CAI-like material can be present in comets, which is interpreted as evidence for radial mixing (Zolensky et al. 2006). In infrared observations of protoplanetary disks there is strong evidence of the existence of crystalline silicates at radii in the disk that are far larger than the radius at which the disk temperatures are high enough for thermal annealing. One idea is that they got there by radial mixing from these hot inner regions to the cooler outer regions (Gail 2001; Bockelee-Morvan 2002).

The process of radial mixing by turbulence can be described by a diffusion equation governed by a diffusion coefficient $D$
(Morfill \& Völk 1984). Turbulence also plays an important role in the theory of coagulation of dust (e.g. Völk et al. 1980). It can both prevent and accelerate grain growth, in complex ways. The process of accretion is, on the other hand, described by the equations of mass- and angular momentum conservation in a viscous medium, which is ruled by the effective viscosity coefficient $v$ (Shakura \& Sunyaev 1973; Lynden-Bell \& Pringle 1974). Both $v$ and $D$ may depend on distance to the star (i.e. the radial coordinate of the disk $R$ ) and both have the same dimension. In fact, because they both arise from the same turbulence, there are reasons to believe that they are nearly the same, apart from a dimensionless factor of order unity which is called the Schmidt number: $v / D \equiv$ Sc. The effective viscosity $v$ may be partly produced by Reynold stress (turbulent motions of the gas), Maxwell-stress (magnetic field lines transporting angular momentum), as well as gravity waves in moderately gravitationally unstable disks. However, the mixing $D$ can only be due to Reynold stress (turbulent motions). So $D$ and $v$ are clearly related, but do not necessarily have to be equal.

As has been shown by Clarke \& Pringle (1988), the efficiency of outward radial mixing in steady disks depends on the ratio of $v$ to $D$, i.e. on $\mathrm{Sc}$, and therefore the Schmidt number plays an essential role in the understanding of the distribution of various chemical and dust species in disks, much more so than the absolute values of $D$ and $v$. The Schmidt number of turbulent flows has been discussed in several studies in the past (e.g. Tennekes \& Lumley 1972; McComb 1990). These authors typically argue for $v / D \simeq 0.7$. It is not clear, however, whether these findings can be directly translated to turbulence in rotating Keplerian accretion disks. Accretion disk flows are fundamentally different from laboratory flow (Balbus 2003). Stone \& Balbus (1996) have shown, using 3D hydrodynamical simulations as well as analytical arguments, that hydrodynamical turbulence resulting from vertical convection tends to transport 
angular momentum inward instead of outward. This stands in contrast to turbulence in planar shear flows which, as Stone \& Balbus confirm in their simulations, transports momentum in the opposite direction. The same kind of inward angular momentum transport was reported by Rüdiger et al. (2005).

Currently the most widely accepted theory for the origin of effective viscosity in accretion disks is that of magnetorotational turbulence. This picture is based on an interplay between weak magnetic fields and the differentially rotating gas in the disk, causing an instability that drives the turbulence (Balbus \& Hawley 1991). Angular momentum is then transported both by Reynold stresses as well as by Maxwell stresses. However, there are various uncertainties about whether the magnetorotational instability can operate in protoplanetary disks which are often very near to being neutral (e.g. Semenov et al. 2004; Ilgner \& Nelson 2006; Oishi et al. 2007). All in all it is still quite unclear what the nature of the turbulence and the mechanism of angular momentum transport is, and therefore the issue of the $v / D$ ratio also remains open.

Recently, several publications have attempted to shed light on the $v / D$ ratio in accretion disks with full 3D MHD simulations of such turbulence. These simulations have yielded values ranging from $v / D \simeq 10$ (Carballido et al. 2005) to $v / D \simeq 0.85$ (Johansen \& Klahr 2005). The drawback of these 3D MHD slab geometry models is that they have a finite numerical resolution which is in general rather coarse. There are reasons to believe that simulations with higher resolution might yield different results. Such initial calculations seem to indeed indicate a reduction of magneto-rotational instability, (Dzyurkevich 2007, private communication).

At the same time, new 2D models of Keller \& Gail (2004) and Tscharnuter \& Gail (2007) show the presence of large-scale circulations within the disk. These calculations support the results found previously in the analytical paper of Urpin (1984), and in the asymptotic study of Regev \& Gitelman (2002). Following these results, beyond the certain critical radius near the disk's equatorial plane, the material moves in the outward direction, whereas the accretion flow develops in the surface layer of the disk. As a result of the large-scale circulation, which is driven by viscous angular momentum transfer, advective transport dominates diffusive mixing in the outer part of the disk. Species that are produced or undergo chemical reactions in the warm inner zones of the disks are advectivelly transported into the cool outer regions. Such dynamics cannot be purely represented by any $1 \mathrm{D}$ viscous or diffusional models. Thus, $v / D$ ratio as direct measure of the mixing efficiency is not appropriate in this picture. But, despite the beauty of this theory, it is based on the postulated anomalous $\alpha$-viscosity, which is an apriory microscale quantity. If strong turbulence exists on the scale which is comparable to the height of the disk, it may destroy such a circulation pattern.

The question is now: which scenario of the disk evolution (viscous, diffusion or advective) is the right one in nature? From theoretical arguments it is still challenging to tell. But we propose here an observational test that may possibly distinguish between all these cases. This allows us to "measure" effective $v / D$ for stars as bright as Herbig Ae stars. Unfortunately this will not work for T Tauri stars and Brown Dwarfs, due to lack of spatial resolution.

This paper is organized as follows. In Sect. 2 we re-derive the Pringle (1981) equation on the basis of a simple diffusion recipe, and we thereby obtain the Schmidt number under the assumption that the simple diffusion recipe describes the true motion of gas parcels in the disk. In Sect. 3 we derive the Schmidt number in a different way, by assuming that each component of the fluid obeys the same equation. We obtain the same Schmidt number as in Sect. 2. In Sect. 4 we review how the Schmidt number affects the abundance of crystalline silicates in the inner disk regions. In Sect. 5 we present radiative transfer results for the mid-infrared spectra of spatially resolved disks, and propose how the study of such spectra might provide insight into what is the value of the Schmidt number in real protoplanetary disks.

In this paper we focus purely on the diffusion of gas or of small particles that are well coupled to the gas. Bigger dust particles (in the size range of $1 \mathrm{~cm}$ or bigger) will decouple from the turbulence and drift inward. This is another process which we do not include in this paper. Also, for simplicity we assume that the disk is vertically averaged and axially symmetric.

\section{The diffusion equation for the Keplerian disk: derivation from a discrete model}

In this section we will re-derive the well known equation of Pringle (1981) for the spreading of the accretion disk. The derivation is based on the idea that the evolution of the disk can be completely described by a special kind of turbulent motion of fluid parcels. In normal turbulence each eddy of the disk has a deviation $\delta v$ from the average velocity of the fluid. These are generally non-Keplerian. But the conservation of angular momentum also prevents these gas parcels from drifting too far inward or outward, unless they exchange angular momentum with neighboring gas parcels, which leads to angular momentum transfer in the wrong direction (see above). However, it is known that weakly magnetized disks are unstable to the magneto-rotational instability (Balbus \& Hawley 1991). Magnetic field lines enable two parcels at different radii to exchange angular momentum "over a distance", if they are thread by the same field lines. We suggest an extremely simplified picture for the motions of these parcels: Consider two parcels, one slowly drifting inward, the other slowly drifting outward. We assume that they both have a locally Keplerian angular velocity at all times. This means that the inward moving parcel loses angular momentum while the other gains angular momentum. We assume that neither parcel has angular momentum exchange with its surroundings. So angular momentum can only be conserved if the loss of angular momentum of the inward moving parcel is compensated by the gain of angular momentum by the outward moving parcel. We invoke an unspecified long-range force that enables this exchange of angular momentum between the two parcels. Physically, the only long-range force that might facilitate this is the Lorenz force, by magnetic fields, so we assume that magnetic fields indeed provide this torque. The accretion and spreading of the disk is, in this picture, nothing other than a material diffusion: Packages of gas being transferred randomly, under the condition of local angular momentum conservation. The radial mixing of a passive tracer in the disk is therefore an integral part of this. If the accretion/spreading of the disk is then viewed in a framework of "viscous disks", then the $v / D$ ratio follows strictly from this derivation.

This picture was also presented in Tutukov \& Pavlyuchenkov (2004), where different types of astrophysical disks are described in frames of non-stationary diffusional models. However, they used only numerical models and did not provide the equation which governs the evolution of such disks.

In this section we will derive these equations and cast them in the standard form of viscous disk equations, which yields the ratio $v / D$. We are fully aware that the simplifications of our 


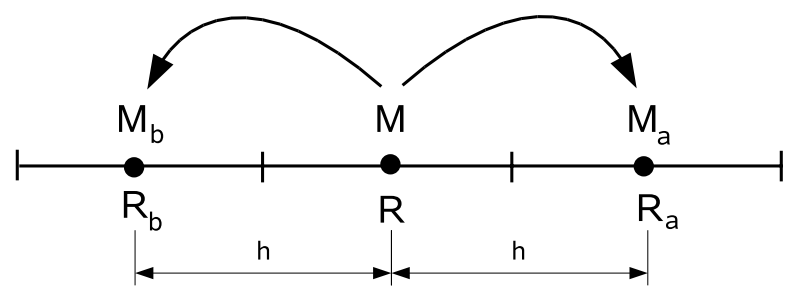

Fig. 1. The scheme of the mass transfer in the model of diffusion disk.

assumptions are quite drastic and that realistic MHD simulations are presumably better and yield somewhat different results. Nevertheless, we justify our approach in light of the fundamental nature of these equations.

\subsection{The mass fluxes from the cell}

Let the mass of the disk be small enough so its self-gravitation can be neglected. The disk is assumed to be geometrically thin, Keplerian, and axially symmetric. We describe the structure of the disk in terms of the surface density $\Sigma(R)$, where $R$ is the distance from the axis of symmetry.

We introduce a grid $\left\{R_{i}\right\}$, which divides the domain into annular elements which form the grid cells. We assume that the cell size $h_{i}=\left(R_{i+1}-R_{i}\right)$ is equal to the characteristic radial mean free path of a turbulent element. The surface density $\Sigma_{i}$ is assumed to be constant within each cell. Along with $\Sigma_{i}$, the average orbital Keplerian velocity is also determined in each grid cell, $V_{i}$, as well as the average radial component of the turbulent velocity, $V_{r, \text { turb }}(i)$.

Let us consider a current cell and denote the nearest cells A and $\mathrm{B}$, see Fig. 1 . We suppose that in a time $\Delta t$ the mass $M$ in the current cell moves to the neighboring cells due to turbulent motion. The matter leaving this cell is transferred to the two adjacent cells, and this redistribution of the mass must conserve mass and angular momentum according to the philosophy described above. The corresponding equations for the system of three cells can be written

$M=M_{\mathrm{a}}+M_{\mathrm{b}}$

$M R V=M_{\mathrm{a}} R_{\mathrm{a}} V_{\mathrm{a}}+M_{\mathrm{b}} R_{\mathrm{b}} V_{\mathrm{b}}$,

where $V_{\mathrm{a}}$ and $V_{\mathrm{b}}$ are the Keplerian velocities for radii $R_{\mathrm{a}}$ and $R_{\mathrm{b}}$, respectively.

Here we will derive the differential equation which describes the evolution of such a disk in the limit $h \rightarrow 0$, assuming also for simplicity that $h_{i}=h=$ const. Using the Taylor expansion of Eq. (2) we obtain (see Appendix A1):

$$
\begin{aligned}
& M_{\mathrm{a}}=\frac{M}{2}\left(1+\frac{1}{4} \frac{h}{R}\right) \\
& M_{\mathrm{b}}=\frac{M}{2}\left(1-\frac{1}{4} \frac{h}{R}\right) .
\end{aligned}
$$

Note that this looks as if mass is always transported outward, since $M_{\mathrm{b}}<M_{\mathrm{a}}$. However, as we shall see below, the true radial flux of matter may have either sign.

\subsection{The mass flux across the cell boundary}

Denote $M_{\mathrm{a}}^{(R)}$ as a mass transferred from the cell with $R$ into the cell $R+h$. Also, let $M_{\mathrm{b}}^{(R+h)}$ be a mass transferred from the

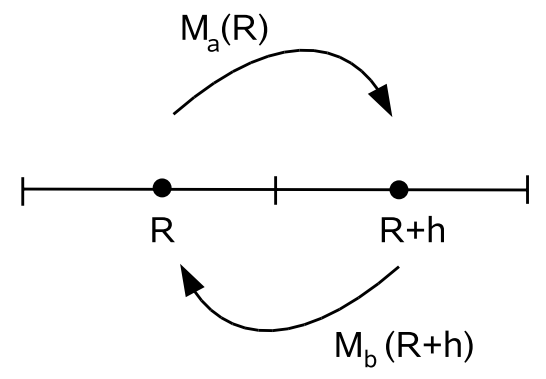

Fig. 2. The scheme of the mass transfer between two cells in the model of diffusion disk.

cell $R+h$ into the cell $R$, see Fig. 2. Denote also $F_{\mathrm{ab}}$ as a total flux across the boundary between the cells:

$F_{\mathrm{ab}} \Delta t=M_{\mathrm{a}}^{(R)}-M_{\mathrm{b}}^{(R+h)}$.

Using the Taylor expansion of Eq. (5) (see Appendix A.2) we find:

$F_{\mathrm{ab}} \Delta t=-\frac{1}{2} h R^{1 / 2} \frac{\partial}{\partial R}\left(M R^{-1 / 2}\right)$.

Note, that if the mass flux $M^{(R)}$ does not depend on radius, then $F_{\mathrm{ab}}>0$, i.e. the mass is transferred outwards.

One of the basic assumptions of our diffusion model is the relation for the flux from the cell:

$M=2 \pi R \cdot \Sigma \cdot V_{r, \text { turb }} \cdot \Delta t$.

If we substitute this relation into Eq. (6) and introduce the diffusion coefficient (see Appendix A3 for the choice of numerical coefficient):

$D=\frac{1}{2} h \cdot V_{r, \text { turb }}$

we get the final formula for the total flux across the cell boundary:

$F_{\mathrm{ab}}=-\pi R^{1 / 2} \frac{\partial}{\partial R}\left(D \Sigma R^{1 / 2}\right)$.

Note now that if $\Sigma$ and $D$ do not depend on radius than $F_{\mathrm{ab}}=$ $-\pi D \Sigma<0$ i.e. the disk becomes an accretion disk.

\subsection{The equation of the diffusion disk evolution}

The law of the mass conservation means:

$2 \pi R \Delta \Sigma=-\frac{\partial F_{\mathrm{ab}}}{\partial R} \Delta t$.

Using Eq. (9) we find finally:

$\frac{\partial \Sigma}{\partial t}=\frac{1}{R} \frac{\partial}{\partial R}\left[\sqrt{R} \frac{\partial}{\partial R}(\Sigma D \sqrt{R})\right]$

This is the required equation which describes the evolution of the diffusive disk.

If we take $v=D / 3$ then we can cast Eq. (11) into the familiar equation for accretion disk evolution (see Pringle 1981):

$\frac{\partial \Sigma}{\partial t}=\frac{3}{R} \frac{\partial}{\partial R}\left[\sqrt{R} \frac{\partial}{\partial R}(\Sigma v \sqrt{R})\right]$.

This equation can be derived from the Navier-Stokes equations for viscous disks, where $v$ is the standard viscosity. Since $D$ is the true diffusion coefficient of the matter, we have found that the ratio $v / D$ must be $1 / 3$ in our picture. 


\section{The diffusion equation for Keplerian disk: multicomponent medium}

Another way to "derive" the ratio of $v / D$ is to start from the Ansatz that each gas or dust species evolves independently according to:

$$
\frac{\partial \Sigma_{i}}{\partial t}=\frac{3}{R} \frac{\partial}{\partial R}\left[\sqrt{R} \frac{\partial}{\partial R}\left(\Sigma_{i} v \sqrt{R}\right)\right]
$$

where $\Sigma_{i}$ is the surface density of species $i$. The $\Sigma_{i}$ obey completeness:

$\Sigma_{1}+\Sigma_{2}+\cdots+\Sigma_{n}=\Sigma$

where $n$ is the total number of species.

The underlying argument to support the Ansatz of Eq. (13) is the following: suppose that gas+dust parcels indeed split up and move inward/outward, as assumed in Sect. 2. The relative abundances of the various species are the same in the inward moving parcel as in the outward moving parcel (they were both from the same original parcel). This means that if the angular momentum stays constant between the inward and outward moving parcel, the same can be said of the individual species. So: species $i$ in the inward moving parcel loses as much angular momentum as the same species in the outward moving parcel gains. Therefore the diffusion happens in each species identically, and therefore we argue that Eq. (13) holds for each species separately, independent of the other species. This is even true if one of the species represents $99.9 \%$ of the mass and the other only $0.1 \%$.

So, starting from Eq. (13) we can rewrite:

$$
\begin{aligned}
\frac{\partial \Sigma_{i}}{\partial t} & =\frac{3}{R} \frac{\partial}{\partial R}\left[\sqrt{R} \frac{\partial}{\partial R}\left(\frac{\Sigma_{i}}{\Sigma} \Sigma v \sqrt{R}\right)\right] \\
& =\frac{3}{R} \frac{\partial}{\partial R}\left[\sqrt{R} \frac{\Sigma_{i}}{\Sigma} \frac{\partial}{\partial R}(\Sigma v \sqrt{R})+R \Sigma v \frac{\partial}{\partial R}\left(\frac{\Sigma_{i}}{\Sigma}\right)\right] .
\end{aligned}
$$

If we write Eq. (12) as a conservation equation as $\dot{\Sigma}+$ $(1 / R) \partial\left[R \Sigma v_{R}\right] / \partial R=0$ where $v_{R}$ is the average radial velocity of the matter (including all species), then by Eq. (12) this velocity is given as:

$v_{R}=-\frac{3}{\Sigma \sqrt{R}} \frac{\partial}{\partial R}(\Sigma v \sqrt{R})$.

Using Eq. (16) in Eq. (15) one obtains:

$\frac{\partial \Sigma_{i}}{\partial t}+\frac{1}{R} \frac{\partial}{\partial R}\left(R \Sigma_{i} v_{R}\right)=\frac{3}{R} \frac{\partial}{\partial R}\left[R v \Sigma \frac{\partial}{\partial R}\left(\frac{\Sigma_{i}}{\Sigma}\right)\right]$.

This is the same as the equation introduced by Morfill \& Völk (1984) but now with:

$\frac{v}{D}=1 / 3$.

So we have shown here that, provided $v / D=1 / 3$, the picture proposed by Morfill \& Völk (1984) that diffusion takes place in the comoving frame of an otherwise laminar accretion disk is equivalent to our picture of exchanging Keplerian parcels.

Again we stress that our derivation is based on a simplifying assumption. One can consider our value of $v / D=0.33$ as a minimum value of $v / D$. At the other extreme is the picture of a perfectly laminar but viscous disk with $v / D=$ inf. The true value must be within these limits.

\section{Effect of $v / D$ on abundance of crystalline silicates in disks}

Although our simple analytic argumentation predicts that $\mathrm{Sc}=$ $1 / 3$, MHD simulations and experiments with rotating turbulent flows show other ratios. At the moment we need to consider values ranging from $\mathrm{Sc}=1 / 3$ (this study) to $\mathrm{Sc}=10$ (Carballido et al. 2005; Johansen et al. 2006). So how do these various values affect the distribution of molecular species and dust species in the disk? What are the consequences of this, for instance, for the problem of crystalline silicates in disks? One may argue that the difference between $v / D=1 / 3$ and $v / D=1$ is "only" a factor of 3 and therefore not very strong: the time scales of diffusion will be different by only a factor of 3 . However, for accretion disks in semi-steady state, a difference of 3 in the diffusion constant can have very strong effects on the distribution of thermally processed (crystalline) particles in the disk. If we assume that particles are only thermally processed in the very inner regions of the disk (i.e. close to the star) then to find particles at larger radii, they have to diffuse outward (e.g. Gail 2001; BockeleeMorvan 2002). The efficiency of this outward diffusion is very dependent on the $v / D$ ratio. This is due to the fact that the trace particles (crystalline dust) have to "swim upstream" against the accretion flow to reach larger radii. This was modeled by Clarke \& Pringle (1988) who showed that the abundance of such a tracer as a function of radius can be derived analytically for steady accretion disks. Since in the absence of disk instabilities the inner regions of viscously evolving disks change over a time scale much larger than the local viscous time, these inner regions (say, inward of a few tens of $\mathrm{AU}$ at an age of $1 \mathrm{Myr}$ ) can be regarded as being in semi-steady state. This simplifies the analysis drastically. We can then start from the standard advection-diffusion equation, after Morfill \& Völk (1984):

$\frac{\partial \Sigma_{i}}{\partial t}+\frac{1}{R} \frac{\partial}{\partial R}\left(R \Sigma_{i} v_{R}\right)=\frac{1}{R} \frac{\partial}{\partial R}\left[R D \Sigma \frac{\partial}{\partial R}\left(\frac{\Sigma_{i}}{\Sigma}\right)\right]$.

For the special case of $v / D=1 / 3$ this becomes equal to Eq. (17), but we will retain the more general form of Eq. (19) here. For a steady state disk we have (for $R \gg R_{\text {in }}$ where $R_{\text {in }}$ is the inner radius of the disk):

$\dot{M}=-2 \pi R \Sigma v_{R}$,

with

$v_{R}=-\frac{3}{2} \frac{v}{R}$

Here $\dot{M}$ is the accretion rate of the disk. With the Schmidt number Sc $\equiv v / D$, by using Eqs. (20)-(21), and by taking $\partial \Sigma_{i} / \partial t=0$ (steady state) we can rewrite Eq. (19) in the form:

$\frac{\partial}{\partial \lg R}\left(\frac{\Sigma_{i}}{\Sigma}\right)=-\frac{2}{3} \frac{1}{\operatorname{Sc}} \frac{\partial^{2}}{\partial(\lg R)^{2}}\left(\frac{\Sigma_{i}}{\Sigma}\right)$.

The solution is:

$\frac{\Sigma_{i}}{\Sigma}=\sigma_{i}^{(0)}+\sigma_{i}^{(1)}\left(\frac{R_{1}}{R}\right)^{\frac{3}{2} \mathrm{Sc}}$

This is a general steady-state solution for mixing of any tracer in the disk for $R \gg R_{\text {in }}$. The $\sigma_{i}^{(0)}$ is a background abundance while $\sigma_{i}^{(1)}$ is the abundance at some radius $R_{1}$.

In general, the Eq. (23) is appropriate only if no large-scale radial flows are present in the disk. However, it is possible to modify this equation in a way that it approximately accounts for 


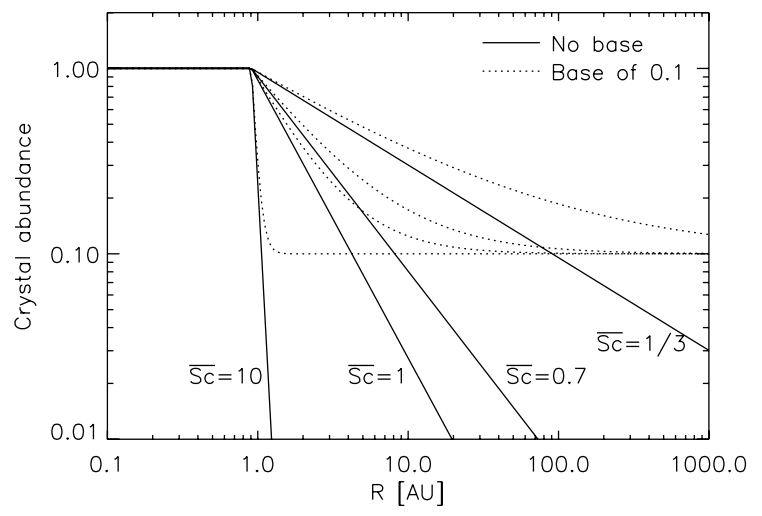

Fig. 3. The abundance of crystalline silicates in a steadily accreting protoplanetary disk around a Herbig star of $M_{*}=2.5 M_{\odot}, R_{*}=2.5 L_{\odot}$ and $T_{*}=10^{4} \mathrm{~K}$. Solid lines: assuming that only thermal annealing in the warm inner regions (here: inward of about $1 \mathrm{AU}$ ) can produce crystalline silicates. Dotted lines: assuming that some other mechanism has produced a global base level of crystallinity of $10 \%$.

the effect of large-scale advective flows onto emergent spectra. Following the results of Keller \& Gail (2004) and Tscharnuter \& Gail (2007) we assume that near the disk's midplane the material moves outward, while in the surface layers the material flows inward. Since we assume that the surface layer of the disk is responsible for the emergent spectra we change the Eq. (23) so as it describes the abundances of the tracers in the surface layer. Therefore, we substitute $V_{R}(1+C)$ for $V_{R}$ in the Eq. (20), where $C$ is the circulation velocity in units of the vertically averaged accretion velocity, and thus we come to a more general relation for the tracer abundances in the surface layer:

$\frac{\Sigma_{i}}{\Sigma}=\sigma_{i}^{(0)}+\sigma_{i}^{(1)}\left(\frac{R_{1}}{R}\right)^{\frac{3}{2} \operatorname{Sc}(1+C)}$.

We suppose that $C$ can easily be $2-3$ or more which has major consequences for the mixing. Given this modification, we introduce the effective Schmidt number:

$\overline{\mathrm{Sc}}=\operatorname{Sc}(1+C)$.

Note, that large values of $C$ "mimic" large Schmidt numbers, Sc, in the picture with no circulation.

Let us now apply these results to the problem of crystallinity of dust in a disk which is hot enough for thermal annealing inward of $R_{\text {anneal }}$. Since the dependence of the efficiency of thermal annealing on temperature is extremely sharp, one can say that inward of $R_{\text {anneal }}$ all dust is crystalline. For $R>R_{\text {anneal }}$ there is no annealing and the mixing solution Eq. (24) is valid in which $R_{1} \equiv R_{\text {anneal }}$ and $\sigma_{\text {cryst }}^{(1)}=1$. The background crystallinity $\sigma_{\text {cryst }}^{(0)}$ is the globally present level of crystallinity, discussed below. One sees that the abundance of crystalline silicates at any given radius depends only on $R_{\text {anneal }}$, the base level of crystallinity $\sigma_{\text {cryst }}^{(0)}$ and the value of Sc. It does not depend on the value of $v$ itself (i.e. not on the value of $\alpha$ ). For strong or weak turbulence the results are the same, as long as Sc remains the same. The solutions to Eq. (24) for $\sigma_{\text {cryst }}^{(0)}=0$ (no global crystallinity) and for $\sigma_{\text {cryst }}^{(0)}=0.1$ (10\% global crystallinity) are shown in Fig. 3.

It is important to keep in mind that these analytic solutions for the level of crystallinity are only valid for $R_{1} \equiv R_{\text {anneal }} \gg R_{\text {in }}$. This is the case for Herbig stars, since they are so bright that the annealing radius is around $1 \mathrm{AU}$ while the inner disk radius is more than hundred times smaller. For T Tauri stars, on the other hand, the annealing radius is only about ten times larger than the inner radius, which means that the effects of the no-friction boundary condition of the disk at $R_{\text {in }}$ are still strongly affecting the solution, and hence the above analytic solution is not completely correct. Nonetheless, the rough form remains correct even in that case.

Now let us focus on the case without a base level of crystallinity. It can be seen that the abundance of crystals at large radii is extremely sensitive to the power index $\overline{\mathrm{Sc}}$. For $\overline{\mathrm{Sc}}=10$, i.e. for large Schmidt numbers or large surface inflow velocity, there is essentially no outward mixing. For $\overline{\mathrm{Sc}}=1$ or $\overline{\mathrm{Sc}}=0.7$ the crystals are mixed out to a level of $3 \%$ and $10 \%$ respectively at $10 \mathrm{AU}$. For $\overline{\mathrm{Sc}}=1 / 3$ the $10 \%$ crystallinity is reached at $100 \mathrm{AU}$, i.e. out to a hundred times larger radius. This shows that the seemingly small differences between the various theoretically derived values of Sc make a very large difference in the distribution of crystalline silicates in the disk.

So far we have assumed that crystalline silicates are only produced by thermal annealing in the inner disk regions $(R \leq$ $\left.R_{\text {anneal }}\right)$. However, there is an on going debate about what causes dust grains to become crystalline. While the thermal annealing and radial mixing of dust (Gail 2001) is a natural phenomenon that is likely to happen in all disks, there may be additional mechanisms of crystallizing dust such as shock heating (Harker \& Desch 2002) or lightning (Gibbard 1997, and references therein). Evidence for these alternative mechanisms is found in infrared spectroscopy of protoplanetary disks, where it is found in many sources that the crystalline grains are dominated by forsterite in the outer regions of many disks. This is contrary to the radial mixing models which predict that enstatite dominates in the outer disks (Bouwman et al., in prep.).

From the solar system there is also some evidence that other mechanisms might crystallize dust or dust aggregates (e.g. review by Trieloff \& Palme 2006). However, the inner regions of disks, near the dust sublimation radius, are always warm enough to assure that thermal annealing takes place. Therefore, independent of the existence of other crystallization processes, we expect with near certainty that inward of some radius all the dust is $100 \%$ crystalline (i.e. $\sigma_{\text {cryst }}^{(0)}+\sigma_{\text {cryst }}^{(1)}=1$ ). The other crystallization processes, assuming that they can crystallize dust throughout the disk, produce a base level $\sigma_{\text {cryst }}^{(0)}$ of crystallinity. The dotted lines in Fig. 3 show how the combined effects of crystallization work out. We assume here that the time scales of these additional crystallizing processes are longer than the local viscous time in the region we study here, so that the level of crystallinity in the outer disk regions has been built up over a long time and remain nearly unchanged on the shorter time scales in this inner region.

Another source of a "base-level" of crystallinity $\sigma_{\text {cryst }}^{(0)}$ is that thermal annealing may also happen throughout the disk in the very early disk-formation phases (Dullemond et al. 2006). They showed that in the stage that the disk is formed, depending on initial conditions, much of the outward transport of material may not be due to the "upstream" diffusion, but due to the fact that the disk material itself is spreading outward (Lynden-Bell \& Pringle 1974). Later, this pre-processed material accretes back inward and forms a base level of crystallinity $\sigma_{\text {cryst }}^{(0)}$ (see Dullemond et al. 2006, Fig. 2).

If large-scale circulations are present in the disk then over a large distance there may be vertical mixing between the outward moving midplane and inward moving surface layers. This is likely to produce an enhanced "base" value of crystallinity as it inserts crystalline grains into the surface layers at large radii. 
The conclusion of this section is that radial mixing can have a strong effect on the distribution of crystalline dust, which is important for understanding infrared observations of protoplanetary disks, as well as for understanding the constituents of meteorites. Radial mixing may also strongly affect disk chemistry or grain growth. A small difference in the assumed value of Sc as well as of $C$ could make a strong difference in the results of the models.

\section{5. "Measuring" Sc from disks around Herbig Ae stars}

It is clear that it is of high importance to understand which is the precise value of $\overline{\mathrm{Sc}}$. Theories will likely always have uncertainties. In this section we propose a way to derive the value of $\overline{\mathrm{Sc}}$ observationally. From Fig. 3 it is clear that for Herbig Ae stars the crystallinity of the dust in the region roughly between $1 \mathrm{AU}$ and $10 \mathrm{AU}$ is strongly affected by the ratio Sc, even if there is a base level $\sigma_{\text {cryst }}^{(0)}$ of crystallinity due to other processes. And in the case of Schmidt numbers below 1 the radial mixing may even affect regions as far as out 100 AU. If we can observationally measure the crystallinity as a function of radius then we may be able to determine both $\overline{\mathrm{Sc}}$ as well as $\sigma_{\text {cryst }}^{(0)}$.

The abundance of crystalline dust in a disk can be determined from the analysis of infrared spectra (see review by Natta et al. 2007). For ground-based observations the $8-13 \mu \mathrm{m}$ window is well-suited for this. There are by now a number of instruments and telescopes that can spectrally and spatially resolve disks in the 10 micron regime at spatial resolutions of about 20 AU for typical sources: e.g. VISIR at the VLT, COMICS at Subaru and T-ReCS at Gemini-South. A spatial resolution of about 1 AU can be obtained with mid-infrared interferometry with the MIDI instrument on the VLT (Leinert et al. 2004). Although in particular the interferometry observations do not directly yield spectra as a function of radius, they do give information on those spatial scales that can be compared to model predictions. Of course these model predictions have to be done for each source individually.

To demonstrate the principle, we show the intensityspectrum at various radii in a model of a disk around a Herbig Ae/Be star of $M_{*}=2.5 M_{\odot}, R_{*}=2.5 L_{\odot}$ and $T_{*}=10^{4} \mathrm{~K}$, i.e. the same system as was shown in Fig. 3. We took a disk with a mass of $0.0015 M_{\odot}$, a size of $90 \mathrm{AU}$ and a surface density profile of $\Sigma(R) \propto R^{-1}$. The 2-D axisymmetric density structure of the model was taken to be $\rho(r, z)=\Sigma(R) \exp \left(-z^{2} / 2 R^{2}\right) /\left(H_{\mathrm{p}}(R) \sqrt{2 \pi}\right)$, where $H_{\mathrm{p}}(R)$ is the pressure scale height estimated from the midplane temperature of a simple flaring disk model of the kind of Chiang \& Goldreich (1997). We included two dust species, both consisting of $0.1 \mu \mathrm{m}$ size grains. The first species is a mixture of $25 \%$ amorphous olivine, $25 \%$ amorphous pyroxene and $50 \%$ carbon. The second species consists of $25 \%$ crystalline enstatite and $25 \%$ crystalline forsterite and $50 \%$ carbon. Within each species the various components are in thermal contact, but the two species are not in thermal contact with each other. We used optical constants from Dorschner et al. (1995) for the amorphous olivine and pyroxene, from Servoin \& Piriou (1973) for crystalline forsterite and from Jäger et al. (1998) for crystalline enstatite. The method of computation of the opacities from these constants is described in Min et al. (2005). Given the density structure, the opacities and the stellar parameters we can apply a Monte-Carlo radiative transfer code (RADMC, see Dullemond \& Dominik 2004) to compute the dust temperature everywhere in the disk. RADMC is a Monte Carlo code for dust continuum radiative transfer based on the method of Bjorkman \& Wood (2001) combined with the method of Lucy (1999). This gives the typical structure of irradiated disks which have a warm surface and a cooler interior. A ray-tracing program is then applied to compute, from these density and temperature structures, a spectrum. Due to the warm surface layer lying on top of a cooler interior, the dust features appear in emission in the spectrum. Figure 4 shows the intensity spectra $\left(I_{\nu}(R)\right)$ obtained in this way at various radii (arbitrarily normalized) for four different values of Sc, assuming that there is no base level of crystallinity, i.e. that thermal annealing in the inner disk regions is the only source of crystallinity. The intensity spectrum is the intensity of the face-on disk image as seen at that particular radius, as a function of wavelength. In Fig. 5 the same is shown, but including a base level $\sigma_{\text {cryst }}^{(0)}$ of crystallinity due to either the disk's formation history (e.g. Dullemond et al. 2006) or due to additional crystallization processes (e.g. Harker \& Desch 2002).

From Figs. 4 and 5 one can see that both the base level $\sigma_{\text {cryst }}^{(0)}$ and the $\overline{S c}$ affect the spectra in a way that would allow the reconstruction of these two values from the observations. The $\sigma_{\text {cryst }}^{(0)}$ is reflected in the fact that the strength of the crystalline features decreases with radius but gets "stuck" at some level which is set by the value of $\sigma_{\text {cryst }}^{(0)}$. The Sc value is reflected in the rapidity by which the crystalline features become weaker with radius as one goes to larger radii. And $R_{\text {anneal }}$ is found as the radius by which the crystallinity suddenly starts to drop. In principle the $R_{\text {anneal }}$ should follow directly from theoretical considerations, as the dust temperature in the disk's surface layer, for a given grain size, can be determined theoretically. However, the grain size is not known beforehand, and an uncertainty of the grain size in the 0.1 to $1 \mu \mathrm{m}$ regime can not be constrained by the analysis of the $10 \mu \mathrm{m}$ feature shape, but it does influence the dust temperature. Therefore an observational determination of $R_{\text {anneal }}$ is necessary and possible.

However, there are some difficulties with this scheme. One obvious problem is that one requires rather accurate measurements of the spectrum at many radii. Since most of the action takes place very close to $1 \mathrm{AU}$, these measurements would rely strongly on the MIDI interferometer at the VLT or its planned successor, and it would require measurements with at least 4 baselines that are all ideally aligned in the same position angle on the sky. Moreover it would be best if the disk is nearly face-on and bright.

Another complication is that thermal annealing may take place in the surface layers at a different radius than the midplane. If the disk's heating is dominated by irradiation, then the midplane temperature of the disk is much smaller than the surface temperature. In this case there are radii where the dust is above annealing temperature in the surface, but below annealing temperature in the disk's midplane. It then depends strongly on the vertical mixing efficiency whether the annealing in the surface can also strongly affect the crystallinity of dust in the midplane. If not, then the $R_{\text {anneal }}$ relevant for the radial mixing might be smaller than the radius where the surface is annealed. One would then expect, as a function of radius, the crystallinity to be unity out to that surface annealing radius and then to suddenly drop to the level of the power-law which originated at a smaller annealing radius. In principle this could also be observed, if sufficiently finely-spaced measurements are taken. If accretional (viscous) heating could heat the disk's midplane sufficiently strongly, then the oppose may be true: the annealing radius would then be larger than the annealing radius computed 


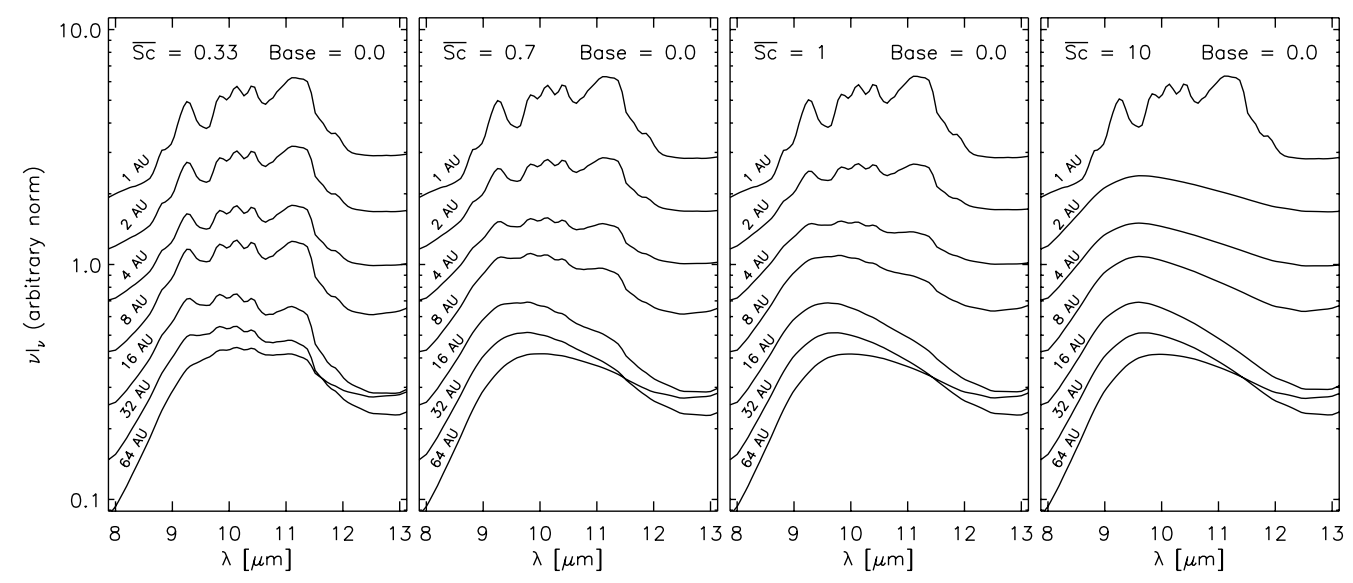

Fig. 4. Intensity spectra of the disk (arbitrarily normalized) at different radii, for different assumed $\overline{\mathrm{Cc}}$, for the standard model shown in Fig. 3. Here the base level of crystallinity $\sigma_{\text {cryst }}^{(0)}$ is chosen to be 0 , i.e. the spectra correspond to the crystallinity levels shown as solid lines in Fig. 3.

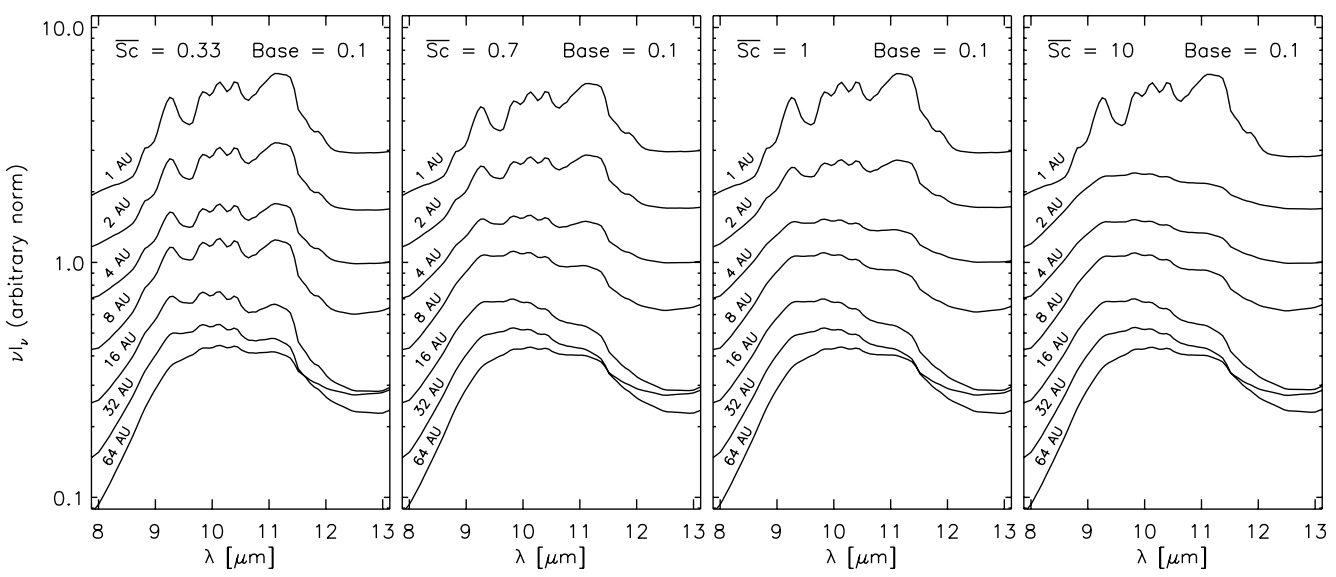

Fig. 5. Same as Fig. 4, but with a base level of crystallinity of $\sigma_{\text {cryst }}^{(0)}=0.1$.

with irradiation only, and only drop at larger radii. Moreover, if meridional flows are present, the picture might blur even more.

It is clear that the full interpretation of these measurements will not be easy. But since the Schmidt number is such a fundamental number for accretion disk theory, it might be worthwhile to attempt to do such measurements nonetheless.

\section{Conclusions}

In this paper we discuss the ratio between turbulent diffusion and viscosity in protoplanetary disks and study an effect of this ratio on the dust crystallinity. Our conclusions can be summarized as follows:

1. The efficiency of radial mixing of any tracers in a steady state disk depends on the ratio between viscosity and diffusion coefficients i.e. on the Schmidt number $\mathrm{Sc}=v / D$ if we assume that the disk is well mixed in vertical direction. Therefore, the Schmidt number plays an essential role in the understanding of the distribution of the various chemical and dust species in disks.

2. We present the simple analytic model of the diffusively spreading disk. We show that evolution of the diffusively spreading disk and evolution of the viscousely spreading disk are described by the same equations if we assume $v / D=1 / 3$ where $v$ is the effective viscosity coefficient in the classical Shakura \& Sunyaev (1973) picture. This does not mean, however, that we necessarily argue that true accretion disks have this $\mathrm{Sc}=1 / 3$ : this depends on the validity of our assumptions (vertically averaged disk, good mixing between dust and gas, a particular kind of turbulence). However, one can regard the value $\mathrm{Sc}=1 / 3$ as the lower limit of the Schmidt number which can be included in the models of radial mixing in Keplerian disks.

3. If large-scale meridional flows are present in the disks then the Schmidt number cannot be used as a direct measure of the mixing efficiency. However, it is possible to approximately describe the abundance of the tracers in the surface layers of the disk by introducing the effective Schmidt number $\overline{\mathrm{Sc}}=\operatorname{Sc}(1+C)$ where $C$ is the layer velocity in units of vertically averaged accretion velocity. This approximation is valid as long as no vertical mixing between in- and outward moving layers is assumed. In this case, we expect less abundance of crystalline dust in the surface layers.

4. We show that the value of $\overline{\mathrm{Sc}}$ may possibly be measured in protoplanetary disks around young stars by using midinfrared interferometry. This requires measurements of a single source at many baselines which all lie along the same position angle across the sky.

Acknowledgements. We are grateful to E. Kurbatov and A.V. Tutukov for help in derivation of the diffusion equation. We are grateful to N. Dzyurkevich for a careful review of the paper. We also wish to thank R. van Boekel, J. Bouwman and M. Min for useful discussions and for the opacity tables. We thank the anonymous referee for very insightful comments which have significantly improved the paper and reminded us of the importance of circulation in disks. 


\section{Appendix A.1}

Consider Eq. (2), and produce the Taylor expansion around central cell, $R$ :

$$
\begin{aligned}
M R V= & M_{\mathrm{a}}(R+h)\left(V+\frac{\partial V}{\partial R} h+\frac{1}{2} \frac{\partial^{2} V}{\partial R^{2}} h^{2}\right) \\
& +M_{\mathrm{b}}(R-h)\left(V-\frac{\partial V}{\partial R} h+\frac{1}{2} \frac{\partial^{2} V}{\partial R^{2}} h^{2}\right) .
\end{aligned}
$$

After elementary operations, using Eq. (1) and dropping terms of order $h^{3}$ we get:

$\left(M_{\mathrm{b}}-M_{\mathrm{a}}\right)=\frac{M h}{2} \frac{\frac{\partial^{2}}{\partial R^{2}}(R V)}{\frac{\partial}{\partial R}(R V)}$.

For the Keplerian disk, $V \sim R^{-1 / 2}$, this relation becomes:

$\left(M_{\mathrm{b}}-M_{\mathrm{a}}\right)=-\frac{M}{4} \frac{h}{R}$.

Using Eq. (1) we obtain:

$M_{\mathrm{a}}=\frac{M}{2}\left(1+\frac{1}{4} \frac{h}{R}\right)$
$M_{\mathrm{b}}=\frac{M}{2}\left(1-\frac{1}{4} \frac{h}{R}\right)$.

\section{Appendix A.2}

Rewrite the fluxes $M_{\mathrm{a}}^{(R)}$ and $M_{\mathrm{b}}^{(R+h)}$ using Eqs. (3) and (4):

$M_{\mathrm{a}}^{(R)}=\frac{1}{2} M^{(R)}\left\{1+\frac{1}{4} \frac{h}{R}\right\}$

$M_{\mathrm{b}}^{(R+h)}=\frac{1}{2} M^{(R+h)}\left\{1-\frac{1}{4} \frac{h}{(R+h)}\right\}$.

where $M^{(R)}$ and $M^{(R+h)}$ are the masses emerging from the cells with radii $R$ and $R+h$ respectively. Denote $F_{\mathrm{ab}}$ as a total flux across the boundary between the cells:

$F_{\mathrm{ab}} \Delta t=M_{\mathrm{a}}^{(R)}-M_{\mathrm{b}}^{(R+h)}$.

If we take the Taylor expansion of Eq. (32) around $R$ and leave only the linear terms in $h$ we get:

$F_{\mathrm{ab}} \Delta t=\frac{h}{2}\left(\frac{1}{2} \frac{M}{R}-\frac{\partial M}{\partial R}\right)$.

It is convenient to rewrite the last equation in the form:

$F_{\mathrm{ab}} \Delta t=-\frac{1}{2} h R^{1 / 2} \frac{\partial}{\partial R}\left(M R^{-1 / 2}\right)$.

\section{Appendix A.3}

Let us comment on an important point in the above derivation. One may argue that the factor $1 / 2$ in Eq. (8) is an arbitrary number. Thus, the factor which appears in the right part of final Eq. (11) (now it is 1) is also arbitrary. However, we support our choice of 1/2 in Eq. (8) based on the following arguments. It is easy to rewrite the derivation for $1 \mathrm{D}$ planar geometry i.e. to obtain the diffusion equation in Cartesian coordinates. In terms of the above equations we should simply set $M_{\mathrm{a}}=M_{\mathrm{b}}=M / 2$ instead of using Eqs. (3)-(4). The Eq. (6) is replaced by

$F_{\mathrm{ab}} \Delta t=-\frac{h}{2} \frac{\partial M}{\partial R}$

and Eq. (7) is rewritten as

$M=\Sigma \cdot V_{r, \text { turb }} \cdot \Delta t$,

where $M$ is the total mass of the current cell. We should substitute these relations into analogue of Eq. (10) for planar geometry

$\Delta R \cdot \Delta \Sigma=-\left(F_{\mathrm{ab}}(R+h)-F_{\mathrm{ab}}(R)\right) \Delta t$.

In this way we obtain the classical equation:

$\frac{\partial \Sigma}{\partial t}=D \frac{\partial^{2} \Sigma}{\partial R^{2}}$,

only if we introduce the diffusion coefficient, $D$, in the form of Eq. (8). In other words, we choose the convention Eq. (8) in such a way that Eq. (11) turns into Eq. (39) in the limit of $R \rightarrow \infty$ and $D=$ const.

\section{References}

Balbus, S. A. 2003, ARA\&A, 41, 555

Balbus, S., \& Hawley, J. 1991, ApJ, 376, 214

Bjorkman, J. E., \& Wood, K. 2001, ApJ, 554, 615

Bockelée-Morvan, D., Gautier, D., Hersant, F., Huré, J.-M., \& Robert, F. 2002, A\&A, 384, 1107

Carballido, A., Stone, J. M., \& Pringle, J. E. 2005, MNRAS, 358, 1055

Chiang, E. I., \& Goldreich, P. 1997, ApJ, 490, 368

Clarke, C. J., \& Pringle, J. E. 1988, MNRAS, 235, 365

Dorschner, J., Begemann, B., Henning, T., Jäger, C., \& Mutschke, H. 1995, A\&A, 300, 503

Dullemond, C. P., \& Dominik, C. 2004, A\&A, 417, 159

Dullemond, C. P., Apai, D., \& Walch, S. 2006, ApJ, 640, L67

Gail, H.-P. 2001, A\&A, 378, 192

Gammie, C. F. 1996, ApJ, 457, 355

Gibbard, S. G., Levy, E. H., \& Morfill, G. E. 1997, Icarus, 130, 517

Harker, D. E., \& Desch, S. J. 2002, ApJ, 565, L109

Ilgner, M., \& Nelson, R. P. 2006, A\&A, 445, 205

Jäger, C., Molster, F. J., Dorschner, J., et al. 1998, A\&A, 339, 904

Johansen, A., \& Klahr, H. 2005, ApJ, 634, 1353

Johansen, A., Klahr, H., \& Mee, A. J. 2006, MNRAS, 370, L71

Keller, Ch., \& Gail, H.-P. 2004, A\&A, 415, 1177

Klerner, S., \& Palme, H. 2000, Meteor. Plan. Sci. Suppl., 35, A89

Leinert, C., van Boekel, R., Waters, L. B. F. M., et al. 2004, A\&A, 423, 537

Lucy, L. B. 1999, A\&A, 344, 282

Lynden-Bell, D., \& Pringle, J. E. 1974, MNRAS, 168, 603

McComb, W. D. 1990, The Physics of Fluid Turbulence (Oxford Science Publications)

Min, M., Hovenier, J. W., \& de Koter, A. 2005, A\&A, 432, 909

Morfill, G. E., \& Voelk, H. J. 1984, ApJ, 287, 371

Natta, A., Testi, L., Calvet, N., et al. 2007, Protostars and Planets V (Tuscon: University of Arizona Press), 767

Oishi, J. S., Mac Low, M.-M., \& Menou, K. 2007 [arXiv:astro-ph/25490]

Pringle, J. E. 1981, ARA\&A, 19, 137

Regev, O., \& Gitelman, L. 2002, A\&A, 396623

Rüdiger, G., Egorov, P., \& Ziegler, U. 2005, Astron. Nachr., 326, 315

Semenov, D., Wiebe, D., \& Henning, T. 2004, A\&A, 417, 93

Semenov, D., Wiebe, D., \& Henning, T. 2006, ApJ, 647, L57

Servoin, J. L., \& Piriou, B. 1973, Phys. Stat. Sol., 55, 677

Shakura, N. I., \& Sunyaev, R. A. 1973, A\&A, 24, 337

Stone, J. M., \& Balbus, S. A. 1996, ApJ, 464, 364

Tscharnuter, W. M., \& Gail H.-P. 2007, A\&A, 463, 369

Tennekes, H., \& Lumley, J. L. 1972, First course in turbulence (Cambridge: MIT Press)

Trieloff, M., \& Palme, H. 2006, in Planet Formation, ed. H. Klahr, \& W. Brandner (Cambridge, UK: Cambridge University Press)

Tutukov, A. V., \& Pavlyuchenkov, Y. N. 2004, Astron. Rep., 48, 800

Voelk, H. J., Jones, F. C., Morfill, G. E., \& Roeser, S. 1980, A\&A, 85, 316

Urpin, V. A. 1984, Astron. Zh., 61, 84

Zolensky, M. E., Zega, T. J., Yano, H., et al. 2006, Science, 314, 1735 